\title{
Lean Body Weight and Metformin Are Insufficient to Prevent Endometrial Hyperplasia in Mice Harboring Inactivating Mutations in PTEN
}

\author{
David A. Iglesias Qian Zhang Joseph Celestino Charlotte C. Sun \\ Melinda S. Yates Rosemarie E. Schmandt Karen H. Lu \\ Department of Gynecologic Oncology and Reproductive Medicine, University of Texas MD Anderson Cancer Center, \\ Houston, TX, USA
}

\section{Key Words \\ Endometrial hyperplasia $\cdot$ Metformin $\cdot$ Mouse models . \\ Obesity · Prevention · Phosphatase and tensin homolog}

\begin{abstract}
Objectives: Obesity is a major risk factor for endometrial cancer. We evaluated whether obesity exacerbates progression of endometrial hyperplasia (EH) using the $\mathrm{PR}^{\mathrm{Cre} /+} \mathrm{PTEN}^{\mathrm{flox} /+}$ mouse model and examined if the type 2 diabetes drug, metformin, could prevent EH. Methods: Twenty obese (PR $\mathrm{Pre} /+$ $\mathrm{PTEN}^{\mathrm{flox} /+}$ ) mice were maintained on a high-fat diet, while 20 lean mice ate a matching low-fat diet. Ten mice from each group received metformin $(1,000 \mathrm{mg} /$ day $)$ in drinking water. Mice were euthanized at 26 weeks. Uterine tissue was scored for degree of EH. Immunohistochemical staining for Ki67 was used to evaluate cellular proliferation. Markers of PI3K/ AKT/mTOR activity were evaluated by immunohistochemistry using activation-specific antibodies. Serum adiponectin was quantified by ELISA. Results: Obesity had no effect on the extent of $\mathrm{EH}$ in $\left(\mathrm{PR}^{\mathrm{Cre} /+}\right.$ PTEN $\left.{ }^{\mathrm{flox} /+}\right)$ mice. While metformin significantly altered circulating adiponectin levels in obese and lean animals, it had no effect on EH. There were no differences in endometrial proliferation as measured by Ki67 staining. Neither obesity nor metformin altered PI3K/AKT/
\end{abstract}

() 2016 S. Karger AG, Basel

E-Mail karger@karger.com www.karger.com/ocl
mTOR activity in these animals. Conclusions: Weight and metformin did not affect the severity of EH resulting from PTEN inactivation. Alternative mouse models of early endometrial cancer are required for preclinical cancer prevention studies.

(c) 2016 S. Karger AG, Basel

\section{Introduction}

Endometrial cancer (EC) is the most common gynecologic malignancy in the US, with an estimated 52,630 new cases diagnosed in 2014 [1]. Endometrial tumors have traditionally been classified into two types [2-4]. Type I or endometrioid endometrial carcinomas, which represent more than $80 \%$ of all EC cases, are associated with obesity, hyperinsulinemia, and prolonged exposure to unopposed estrogen. These tumors are thought to arise from the progression of endometrial hyperplasia $(\mathrm{EH})$ to complex atypical hyperplasia to well-differentiated endometrial carcinomas (EC). Type II or nonendometrioid endometrial carcinomas, include uterine serous carcinomas, and are typically more aggressive and estrogen independent. Recently, based on extensive genomic analyses of both type I and type II tumors, ECs have been further

Dr. Rosemarie E. Schmandt

Department of Gynecologic Oncology and Reproductive Medicine, Unit 1362 University of Texas MD Anderson Cancer Center, PO Box 301439 Houston, TX 77230-1439 (USA)

E-Mail rschmand@mdanderson.org 
reclassified into multiple subtypes [2, 5-8]. Indeed, the detailed characterization of molecular alterations underlying the pathogenesis of the different histologies of EC lays the groundwork for rational disease prevention strategies and therapeutic drug design [2, 5-8].

The most frequently observed genetic mutations in type I EC occur in the phosphatase and tensin homo$\log$ (PTEN) tumor suppressor gene. PTEN mutation is thought to be an early event in the pathogenesis of EC, and its incidence increases with disease progression [9]. Up to $20 \%$ of cases of EH harbor mutations in PTEN, while over $80 \%$ of type I ECs possess inactivating mutations in PTEN [10]. PTEN acts as both a protein and lipid phosphatase $[11,12]$. It preferentially dephosphorylates phosphatidylinositol-3,4,5-trisphosphate (PIP3), thereby acting in opposition to phosphatidylinositol-4,5bisphosphate 3-kinase (PI3K). Mutations resulting in the loss of function or expression of the PTEN protein, are associated with hyperactivity of the PI3K/AKT/mTOR pathway, thereby increasing cellular proliferation and survival, metastasis and angiogenesis, changes in metabolism and resistance to chemotherapeutic agents [1113].

Genetically modified PTEN 'knockout' mice represent a well-characterized animal model for the study of the progression of EH to type I EC. Without fail, all female mice heterozygous for inactivating mutations in PTEN develop EH by 26 weeks of age, with $20 \%$ of mice progressing to low grade EC by 40 weeks [14-16]. This effect is observed in mouse models with systemic heterozygous disruption of PTEN $\left(\mathrm{PTEN}^{+/}\right)$and in models targeting heterozygous disruption to the endometrium using a Cre-Lox system $\left(\mathrm{PR}^{\mathrm{Cre} /+} \mathrm{PTEN}^{\text {flox/+ }}\right)$. We previously demonstrated that everolimus (RAD001), an mTOR inhibitor, prevented the progression of $\mathrm{EH}$ in these animals [17], indicating that this animal model might be useful to study the chemoprevention of $\mathrm{EH}$.

Obesity greatly increases the risk for EC [3]. The American Institute for Cancer Research estimates that $59 \%$ of ECs can be prevented by maintaining a healthy weight and by being physically active [18]. $\mathrm{PR}^{\mathrm{Cre} /+}$ $\mathrm{PTEN}^{\text {flox/+ }}$ mice bred on a C57BL/6 background are susceptible to diet-induced obesity, with accompanying mild to moderate hyperglycemia and hyperinsulinemia, which mimic the human condition [19-21]. Given the role of obesity in type-1 EC risk, we sought to determine if obesity would accelerate the onset of $\mathrm{EH}$ and its progression to EC in the $\mathrm{PR}^{\mathrm{Cre} /+} \mathrm{PTEN}^{\text {flox/+ }}$ mouse model.

Metformin, a biguanide drug commonly used to treat type 2 diabetes, has recently been shown to exert growth- inhibitory effects for a variety of cancers, including EC both in vitro and in vivo [22-25]. Recent studies by our own group have demonstrated endometrial responsiveness to metformin therapy in a rat model $[24,26]$. Metformin decreases cellular proliferation by multiple mechanisms. Metformin increases insulin receptor sensitivity and decreases circulating insulin levels, thereby modulating insulin receptor-mediated growth. Metformin also activates the growth-inhibitory AMP-dependent kinase (AMPK) pathway, which counteracts signaling through both the PI3K/AKT and MAPK pathways downstream of a variety of growth factor receptors $[22,27]$. Therefore, in addition to examining the effects of obesity on the pathogenesis of $\mathrm{EH}$, we further investigated whether metformin could act as a chemopreventive agent in $\mathrm{PR}^{\mathrm{Cre} /+} \mathrm{PTEN}^{\text {flox/+ }}$ mice whether lean or obese.

\section{Materials and Methods}

\section{Animals}

All procedures described were performed following approval by the MD Anderson Cancer Center Institutional Animal Care and Use Committee, and in adherence to the institutional guidelines. $\mathrm{PR}^{\text {Cre/+ }} \mathrm{PTEN} \mathrm{N}^{\text {flox/flox }}$ mice were obtained from Dr. F. DeMayo, Baylor College of Medicine [28, 29]. Cre recombinase is expressed under the regulation of the progesterone receptor promoter [28]; therefore, in a floxed PTEN female mouse, PTEN is selectively disrupted in PR-expressing tissues, including the endometrium, oviduct, ovary, mammary gland, and pituitary [28]. Male $\mathrm{PR}^{\mathrm{Cre} /+} \mathrm{PTEN}^{\text {flox/flox }}$ which demonstrate no obvious phenotype, were crossed with wild-type C57BL/6 females to generate $\mathrm{PR}^{\mathrm{Cre} /+} \mathrm{PTEN}^{\text {flox/+ }}$ mice. At 6 weeks of age, female mice were divided into two treatment groups $(n=20 /$ group). Twenty mice were fed a high-fat diet (HFD) in which $60 \%$ of calories were obtained from fat (D12492 Research Diets Inc., New Brunswick, NJ) while 20 mice were fed a matching, low-fat diet (LFD; D12450B). Mice were weighed weekly to monitor weight gain. At 10 weeks, when all HFD animals had reached a weight $20 \%$ greater than that of LFD controls, mice were again divided into two treatment groups $(\mathrm{n}=10)$. Ten HFD and 10 LFD mice received metformin $(5 \mathrm{mg} / \mathrm{ml})$ in their drinking water, while controls received untreated water. Animals continued with their respective diets. Both groups drank the same volume of liquid daily, with metformintreated mice receiving a dose of approximately $1,000 \mathrm{mg} / \mathrm{kg}$ daily. All animals were euthanized at 26 weeks of age, when $100 \%$ of lean, untreated controls were expected to develop EH (control group). Serum and uterine tissue were collected for further evaluation.

\section{Scoring for $E H$}

Murine endometrial tissues were evaluated for $\mathrm{EH}$ as previously described [17]. Briefly, hematoxylin and eosin-stained sections of endometrium were reviewed, to determine hyperplasia and grade by a gynecologic pathologist (R.R.B.) who was blinded 
Table 1. The effects of obesity and metformin on degree of endometrial hyperplasia and proliferative signaling in obese and lean $\mathrm{PR}$ Cre/+ $\mathrm{PTEN}^{\mathrm{f} / \mathrm{f}}$ mice

\begin{tabular}{|c|c|c|c|c|c|c|c|c|c|c|c|c|c|c|c|}
\hline & \multicolumn{3}{|c|}{ LFD control } & \multicolumn{4}{|c|}{ LFD + metformin } & \multicolumn{4}{|c|}{ HFD } & \multicolumn{4}{|c|}{ HFD + metformin } \\
\hline & $\mathrm{n}$ & mean & SD & $\mathrm{n}$ & mean & SD & $\mathrm{p}$ & $\mathrm{n}$ & mean & SD & $\mathrm{p}$ & $\mathrm{n}$ & mean & $\mathrm{SD}$ & $\mathrm{p}$ \\
\hline Hyperplasia, score & 10 & 4.70 & 1.89 & 10 & 5.90 & 2.47 & 0.54 & 10 & 5.90 & 2.64 & 0.54 & 10 & 6.10 & 2.38 & 0.42 \\
\hline \multicolumn{16}{|l|}{ Tissue biomarkers } \\
\hline Ki67, \% positive & 10 & 21.2 & 14.6 & 10 & 29.3 & 18.9 & 0.67 & 10 & 30.5 & 20.3 & 0.57 & 10 & 29.0 & 21.8 & 0.69 \\
\hline pS6rp, IHC score & 10 & 5.40 & 2.12 & 10 & 5.00 & 2.11 & 0.98 & 10 & 5.40 & 3.50 & 1.0 & 10 & 4.30 & 3.16 & 0.71 \\
\hline pACC, IHC score & 10 & 2.40 & 0.52 & 10 & 2.40 & 0.52 & 1.0 & 10 & 2.30 & 0.48 & 0.95 & 10 & 2.50 & 0.53 & 0.95 \\
\hline Caspase 3 , IHC score & 10 & 4.70 & 7.85 & 10 & 4.75 & 5.28 & 1.0 & 10 & 6.65 & 6.46 & 0.88 & 10 & 7.65 & 8.76 & 0.69 \\
\hline pERK, IHC score & 10 & 7.00 & 2.58 & 10 & 8.00 & 3.50 & 0.75 & 10 & 7.00 & 2.11 & 1.0 & 10 & 6.00 & 2.73 & 0.75 \\
\hline pAKT, IHC score & 10 & 1.60 & 0.52 & 10 & 1.80 & 0.42 & 0.78 & 10 & 1.80 & 0.79 & 0.78 & 10 & 1.50 & 0.53 & 0.96 \\
\hline \multicolumn{16}{|l|}{ Serum biomarkers } \\
\hline Adiponectin, ng/ml & 10 & 14.5 & 2.41 & 9 & 10.5 & 1.96 & 0.002 & 8 & 15.4 & 3.22 & 0.78 & 8 & 10.9 & 1.40 & $\begin{array}{l}0.007 \\
0.03^{\mathrm{a}}\end{array}$ \\
\hline
\end{tabular}

Unless otherwise noted, all comparisons are made with the LFD control animals. ${ }^{\text {a }}$ Adiponectin levels were significantly reduced in the HFD + metformin group when compared to HFD alone, thereby confirming a systemic metformin response in obese animals.

to the study groups. The description of the hyperplastic lesions in the PTEN heterozygote has not been consistent in previous reports $[14,15,30,31]$. In contrast to human $\mathrm{EH}$ that progresses from simple hyperplasia without atypia to complex hyperplasia with atypia, mouse $\mathrm{EH}$, even in very early hyperplasia, displays cytologic atypia. Therefore, to have a more consistent and quantitative description, we defined 3 grades of hyperplasia according to the percentage and size of the hyperplastic lesions. A score describing the extent of hyperplasia was as follows: $1=<33 \%$ hyperplasia; $2=$ $33-66 \%$ hyperplasia; $3=>66 \%$ hyperplasia. Severity of hyperplastic lesions was scored as follows: (1) the hyperplastic glands were small and regular; (2) mix of small and large size; (3) more than one half of the hyperplastic glands were large and irregular. The overall score for each case was defined by the product of the extent of hyperplasia score and score for severity of the lesion. The hyperplastic lesion was defined as grade 1 when the product of the 2 scores was $1-3$, as grade 2 when the product was $4-6$, and as grade 3 when the product was 7-9.

\section{Immunohistochemical Staining of Endometrial Tissues}

The effect of obesity and metformin on mouse endometrial proliferation was evaluated in formalin-fixed, paraffin embedded tissue by Ki67 staining (BD Biosciences, San Diego, CA, USA) following the manufacturer's instructions. The degree of apoptosis in endometrial tissues was determined by immunohistochemistry (IHC) staining for cleaved caspase-3 (Asp175; Cell Signaling, Danvers, MA, USA). The sections were counterstained with Mayer's hematoxylin. The average number of positively-stained Ki67 or caspase- 3 cells in 5-10 high-power microscopic fields were counted per slide and calculated as: $200 \times$ (number of positively stained endometrial cells/total endometrial cells). Tissues were also stained for downstream markers of insulin and IGF-1 receptor signaling, including phospho-S6 ribosomal protein (Ser235/236), phospho-AKT (Ser473), phospho-p44/42 MAPK (Thr202/Tyr204), and phosphoacetyl-CoA carboxylase (Ser79) (Cell Signaling, Danvers, MA, USA) and scored according to previously published procedures [24].

PTEN Inactivation Confers Resistance to

Cancer-Preventive Strategies

\section{Serum Analyses}

Serum adiponectin levels were evaluated using the Mouse Adiponectin ELISA Kit (Millipore, Billerica, MA, USA). Serum samples were tested in triplicate.

\section{Statistical Analysis}

For each of the following tissue and serum markers: Ki67, pS6rp (phospho-S6 ribosomal protein), pACC (phospho-acetylCoA carboxylase), caspase 3, pERK (phospho-p44/42 ERK), pAKT (phospho-AKT), and adiponectin, a one-way analysis of variance was calculated. Dunnett's test was used to conduct post hoc comparisons of the LFD group (control) with the experimental groups (diet and metformin). All tests were two-sided, and $\mathrm{p}<0.05$ was considered to be statistically significant.

\section{Results}

\section{LFD and Metformin Are Insufficient to Decrease the}

Extent of EH in $\mathrm{PR}^{\mathrm{Cre} /+} \mathrm{PTEN} \mathrm{Nlox} /+^{\text {Mice }}$

Histological scores between treatment groups and the control group were not statistically different (fig. 1; table 1). Mean hyperplasia scores were 4.7, 5.9, 5.9, and 6.1, for control lean mice, obese mice, and metformin-treated lean and obese mice, respectively, with the majority of mice presenting with grade 2 hyperplasia as defined by our scoring system at 26 weeks, irrespective of treatment group. The effect of metformin and diet/obesity on endometrial proliferation was compared using IHC staining for Ki67. Despite a significant weight difference in the HFD versus LFD groups (fig. 1a), no significant differences in $\mathrm{EH}$ were observed at 26 weeks (fig. 1b). Further- 


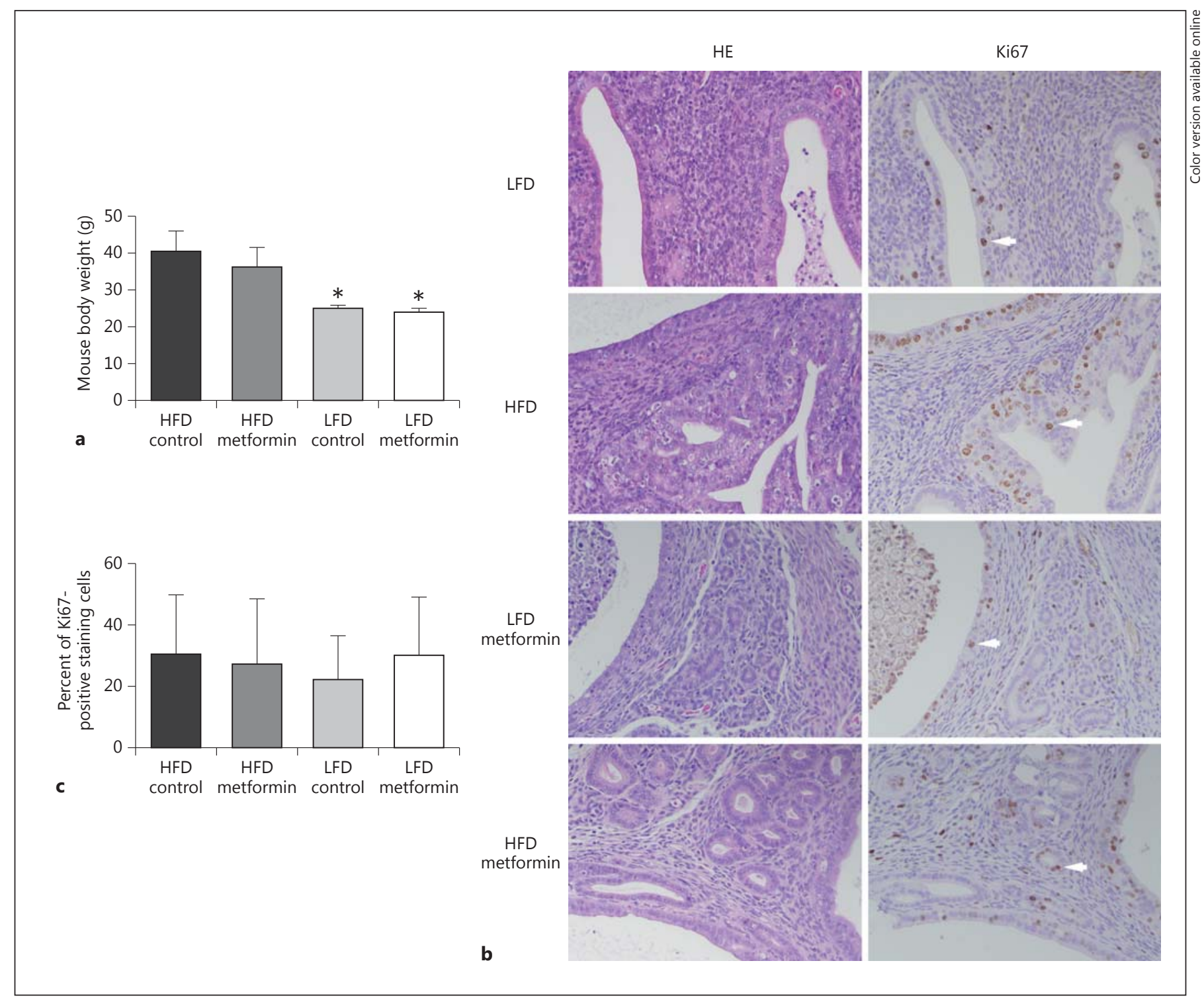

Fig. 1. Obesity and metformin treatment have no detectable effect on the extent of EH in the $\mathrm{PR}^{\mathrm{Cre} /+} \mathrm{PTEN}^{\text {flox/+ }}$ mouse model. a $\mathrm{PR}^{\mathrm{Cre} /+} \mathrm{PTEN}^{\text {flox/+ }}$ mice were fed either an HFD or LFD and either untreated or treated with metformin $(1,000 \mathrm{mg} / \mathrm{kg})$ in drinking water. Animals were weighed and euthanized at 26 weeks. Serum and reproductive organs were collected for analysis. LFD-fed mouse weights, either treated or untreated with metformin, were significantly lower than those in HFD-fed mice. There were no

more, metformin produced no significant changes in animal weight or on the development of $\mathrm{EH}$ in either obese or lean animals. Taken together, no significant difference in hyperplasia score or endometrial proliferation was observed between any of the four treatment groups (fig. 1; table 1). significant changes in weight in response to metformin in either HFD- or LFD-fed animals. b Endometrial tissue was evaluated for the presence of hyperplasia following HE staining, and cellular proliferation was analyzed by IHC staining for Ki67. c No significant difference in cell proliferation was observed between treatment groups, as determined by percentage of Ki67-positive cells in the endometrium.

Markers of Cellular Proliferation and Insulin/IGF-1 Signaling Are Unaffected by either Diet-Induced Obesity or Metformin in the Endometrium of $P R^{C r e /+}$ PTEN flox/+ Mice

Changes in signaling pathways typically affected by obesity and metformin were evaluated in the endome- 
trium by IHC staining using activation-specific antibodies. Findings are summarized in table 1 . No significant differences in IHC staining scores for pS6rp (Ser235/236), pAKT (Ser473), pERK (Thr202/Tyr204), and pACC (Ser79) were observed between the endometrial tissues of any of the three treatment groups as compared to the LFD control group. Furthermore, no differences in apoptosis, as measured by caspase $3 \mathrm{IHC}$ staining, were observed between control lean mice, obese mice, and metformintreated lean and obese mice.

\section{Metformin Suppresses Adiponectin Expression in Both} Obese and Lean $\mathrm{PR}^{\mathrm{Cre} /+} \mathrm{PTEN}^{\text {flox/++}}$ Mice

Recent studies by our group have demonstrated endometrial responsiveness to metformin therapy in a rat model [24]. The apparent lack of effect on signal transduction in the endometrium of $\mathrm{PR}^{\mathrm{Cr} /+} \mathrm{PTEN}^{\text {flox/+ }}$ mice led us to examine whether this animal model was systemically resistant to the effects of metformin.

Insulin levels could not be used as a marker for metformin response as animals in this study were not fasted; however, Huypens et al. [32] observed that metformin reduces the expression of adiponectin expression and release in mouse (3T3-L1) adipocytes. We therefore tested the serum levels of adiponectin as a surrogate marker of response to metformin (table 1). As compared to control lean mice $(14.5 \mathrm{ng} / \mathrm{ml})$, adiponectin levels decreased significantly with metformin treatment in both lean (10.5 $\mathrm{ng} / \mathrm{ml}, \mathrm{p}=0.002)$ and obese $(10.9 \mathrm{ng} / \mathrm{ml}, \mathrm{p}=0.007)$ mice. These findings confirm that $\mathrm{PR}^{\mathrm{Cre} /+} \mathrm{PTEN}^{\text {flox/+ }}$ mice do have a systemic response to metformin, despite the apparent lack of response to this drug in endometrial tissue.

\section{Discussion}

Inactivating mutations in the PTEN tumor suppressor gene, are commonly observed in type I EC, and are thought to represent an early event in the pathogenesis of this disease. In support of this hypothesis, $100 \%$ of female mice heterozygous for inactivating mutations in PTEN develop EH by 26 weeks of age, with $20 \%$ of these mice progressing to grade 1 EC by 40 weeks of age. Our previous studies have demonstrated that PTEN heterozygous mice represent a useful model for the preclinical evaluation of chemotherapeutic drugs, such as everolimus, which may prevent the progression from EH to EC. The findings presented in the current study, however, suggest that this model may be less useful in the evaluation of behavioral therapies, such as diet and exercise, or drugs

PTEN Inactivation Confers Resistance to

Cancer-Preventive Strategies such as metformin, which are intended to prevent or reverse the earliest transformation from normal to hyperplastic endometrium.

In the $\mathrm{PR}^{\mathrm{Cre} /+} \mathrm{PTEN}^{\text {flox/+ }}$ mouse model, diet-induced obesity did not increase the degree of $\mathrm{EH}$ as compared to lean animals fed an LFD. There was no significant difference in Ki67 staining, which is a tissue marker of proliferation. Signaling through the PI3K/AKT and MAPK pathways was unaffected by obesity in endometrial tissue possessing a genetic mutation of PTEN. Furthermore, despite influencing systemic levels of adiponectin, the antidiabetic drug metformin had no apparent effect on $\mathrm{EH}$ in either obese or lean $\mathrm{PR}^{\mathrm{Cre} /+}$ PTEN ${ }^{\text {flox/+ }}$ mice. Taken together, these data suggest that a genetically acquired PTEN mutation in the endometrium was sufficient to drive the development of $\mathrm{EH}$, and that these lesions are resistant to the effects of obesity and metformin.

We have previously demonstrated that everolimus (RAD001) inhibits the progression of EH in PTEN knockout mice. PTEN is a physiological inhibitor of PI3K/ AKT/mTOR pathway. Everolimus (RAD001) specifically inhibits mTOR kinase activity, and inhibits proliferation, even in the absence of PTEN. Our current studies suggest that systemic changes in proliferative signaling in response to diet/exercise/metformin, are insufficient to inhibit PI3K/AKT/mTOR signaling and cannot overcome the progression of $\mathrm{EH}$ driven by germline mutations in PTEN. It is important to note that Fyles et al. [33] have shown that progestin treatment does not prevent endometrial neoplasia in $\mathrm{PTEN}^{+/-}$mice. The lack of hormone effect is also seen in ovariectomized animals, suggesting that progression of $\mathrm{PTEN}^{+/-}$-associated hyperplasia is not driven by estrogen. Joshi et al. [34] have confirmed these findings and demonstrated that endometrial tumorigenesis occurs independently of estrogen or estrogen receptors in the PTEN ${ }^{+/-}$mouse model. And while Kim et al. [35] demonstrated a decrease in overall uterus and tumor size in progesterone-treated, ovariectomized $\mathrm{PR}^{\mathrm{Cre} /+}$ PTEN $^{\mathrm{f} / \mathrm{f}}$ (homozygous loss of PTEN) animals, progesterone ultimately did not prevent cancer in these animals. It is essential to consider these observations in determining the use of this model for translational research questions, as up to $80 \%$ of early $\mathrm{EH}$ are responsive to hormonal therapy in humans [36].

We conclude that, while the PTEN heterozygous knockout mouse model remains useful for the preclinical evaluation of interventions for Cowden disease-associated EC and for treatment of sporadic EC driven by PTEN mutations, it is not suitable for all studies directed at the chemoprevention of early EC. 


\section{Acknowledgments}

We thank Dr. Franco DeMayo for the use of his $\mathrm{PR}^{\mathrm{Cre} /+}$ PTEN $^{\text {flox/+ }}$ mouse model of endometrial cancer. We also thank Dr. Russell Broaddus for his assistance in the evaluation of endometrial hyperplasia in our mice. This project was supported in part by Grant Number P50CA098258 from the National Cancer Institute, and also in part by the National Institutes of Health through MD Anderson's Cancer Center Support Grant CA016672. This article is dedicated to the memory of Dr. Mike Milam, whose studies using the PTEN model of endometrial hyperplasia continue to fuel ongoing research in our lab.

\section{Disclosure Statement}

The authors report no conflict of interest.

\section{References}

1 Siegel R, et al: Cancer statistics, 2014. CA Cancer J Clin 2014;64:9-29.

2 Murali R, Soslow RA, Weigelt B: Classification of endometrial carcinoma: more than two types. Lancet Oncol 2014;15:e268-e278.

3 Schmandt RE, et al: Understanding obesity and endometrial cancer risk: opportunities for prevention. Am J Obstet Gynecol 2011; 205:518-525.

4 Setiawan VW, et al: Type I and II endometrial cancers: have they different risk factors? J Clin Oncol 2013;31:2607-2618.

5 Hong B, Le Gallo M, Bell DW: The mutational landscape of endometrial cancer. Curr Opin Genet Dev 2015;30C:25-31.

6 Cancer Genome Atlas Research Network: Integrated genomic characterization of endometrial carcinoma. Nature 2013;497:67-73.

7 Liu Y, Broaddus RR, Zhang W: Identifying aggressive forms of endometrioid-type endometrial cancer: new insights into molecular subtyping. Expert Rev Anticancer Ther 2015; $15: 1-3$.

8 Liu Y, et al: Clinical significance of CTNNB1 mutation and Wnt pathway activation in endometrioid endometrial carcinoma. J Natl Cancer Inst 2014;106: pii: dju245.

9 Maxwell GL, et al: Mutation of the PTEN tumor suppressor gene in endometrial hyperplasias. Cancer Res 1998;58:2500-2503.

10 Mutter GL, et al: Altered PTEN expression as a diagnostic marker for the earliest endometrial precancers. J Natl Cancer Inst 2000;92: 924-930.

11 Worby CA, Dixon JE: Pten. Annu Rev Biochem 2014;83:641-669.

12 Song MS, Salmena L, Pandolfi PP: The functions and regulation of the PTEN tumour suppressor. Nat Rev Mol Cell Biol 2012;13: 283-296.

13 Rodon J, et al: Development of PI3K inhibitors: lessons learned from early clinical trials. Nat Rev Clin Oncol 2013;10:143-153.

14 Stambolic V, et al: High incidence of breast and endometrial neoplasia resembling human Cowden syndrome in pten ${ }^{+/}$mice. Cancer Res 2000;60:3605-3611.
15 Podsypanina K, et al: Mutation of Pten/ Mmacl in mice causes neoplasia in multiple organ systems. Proc Natl Acad Sci USA 1999; 96:1563-1568.

16 Carnero A, Paramio JM: The PTEN/PI3K/ AKT pathway in vivo, cancer mouse models. Front Oncol 2014;4:252.

17 Milam MR, et al: Reduced progression of endometrial hyperplasia with oral mTOR inhibition in the Pten heterozygote murine model. Am J Obstet Gynecol 2007;196:247.e1-5.

18 World Cancer Research Fund International: Continuous Update Project Report: Food, Nutrition, Physical Activity and the Prevention of Endometrial Cancer. London, WCRF International, 2013.

19 Collins S, et al: Genetic vulnerability to dietinduced obesity in the C57BL/6J mouse: physiological and molecular characteristics. Physiol Behav 2004;81:243-248.

20 Montgomery MK, et al: Mouse strain-dependent variation in obesity and glucose homeostasis in response to high-fat feeding. Diabetologia 2013;56:1129-1139.

21 Almind K, Kahn CR: Genetic determinants of energy expenditure and insulin resistance in diet-induced obesity in mice. Diabetes 2004; 53:3274-3285.

22 Pernicova I, Korbonits M: Metformin - mode of action and clinical implications for diabetes and cancer. Nat Rev Endocrinol 2014;10:143156.

23 Quinn BJ, et al: Repositioning metformin for cancer prevention and treatment. Trends Endocrinol Metab 2013;24:469-480.

24 Zhang Q, et al: Chemopreventive effects of metformin on obesity-associated endometrial proliferation. Am J Obstet Gynecol 2013;209: 24.e1-24.e12.

25 Iglesias DA, et al: Another surprise from metformin: novel mechanism of action via K-Ras influences endometrial cancer response to therapy. Mol Cancer Ther 2013;12:28472856.
26 Zhang Q, et al: CGRRF1 as a novel biomarker of tissue response to metformin in the context of obesity. Gynecol Oncol 2014;133:83-89.

27 Rena G, Pearson ER, Sakamoto K: Molecular mechanism of action of metformin: old or new insights? Diabetologia 2013;56:18981906.

28 Soyal SM, et al: Cre-mediated recombination in cell lineages that express the progesterone receptor. Genesis 2005;41:58-66.

29 Daikoku T, et al: Conditional loss of uterine Pten unfailingly and rapidly induces endometrial cancer in mice. Cancer Res 2008; 68: 5619-5627.

30 Podsypanina K, et al: An inhibitor of mTOR reduces neoplasia and normalizes p70/S6 kinase activity in Pten ${ }^{+/-}$mice. Proc Natl Acad Sci USA 2001;98:10320-10325.

31 Wang $\mathrm{H}$, et al: DNA mismatch repair deficiency accelerates endometrial tumorigenesis in Pten heterozygous mice. Am J Pathol 2002; 160:1481-1486.

32 Huypens P, et al: Metformin reduces adiponectin protein expression and release in 3T3L1 adipocytes involving activation of AMP activated protein kinase. Eur J Pharmacol 2005;518:90-95.

33 Fyles A, et al: Neither ovariectomy nor progestin treatment prevents endometrial neoplasia in pten $^{+/}$mice. Gynecol Oncol 2008; 108:395-401.

34 Joshi A, et al: Endometrial tumorigenesis in $\mathrm{Pten}^{+/-}$mice is independent of coexistence of estrogen and estrogen receptor alpha. Am J Pathol 2012;180:2536-2547.

$35 \mathrm{Kim}$ HI, et al: Steroid hormone intervenes in the endometrial tumorigenesis of pten ablation. J Cancer Prev 2013;18:313-321.

36 Westin SN, Sun C, Broaddus R, Pal N, et al: Prospective phase 2 trial of the Levonorgestrel Intrauterine System (Mirena) to treat complex atypical hyperplasia and grade 1 endometrioid endometrial cancer. Gynecol Oncol 2012;125:S9. 